\title{
Análise do conhecimento dos profissionais de saúde sobre o uso de oxigenoterapia em um hospital universitário de Fortaleza-CE
}

\section{Analysis of knowledge of health professionals on oxygen use in a university hospital Fortaleza-CE}

Patriciane Hedwiges Barreto ${ }^{1}$. Francisca Soraya Lima Silva ${ }^{1}$. Renata dos Santos de Vasconcelos ${ }^{2}$. Raquel Pinto Sales ${ }^{3}$. Thiago Brasileiro de Vasconcelos ${ }^{4}$. Andréa da Nóbrega Cirino Nogueira ${ }^{5}$. Marcelo Alcantara Holanda ${ }^{6}$.

1 Fisioterapeuta, Residente em Terapia Intensiva no Hospital Universitário Walter Cantídio (HUWC), Universidade Federal do Ceará (UFC), Fortaleza, Ceará, Brasil. 2 Fisioterapeuta, doutoranda em Ciências Médicas pela Universidade Federal do Ceará (UFC), coordenadora das UTI's do Hospital Universitário Walter Cantídio (HUWC), Empresa Brasileira de Serviços Hospitalares (EBSERH), Fortaleza, Ceará, Brasil. 3 Fisioterapeuta pela Empresa Brasileira de Serviços Hospitalares (EBSERH), Mestre em ciências médicas pela Universidade Federal do Ceará (UFC), Fortaleza, Ceará, Brasil. 4 Fisioterapeuta, doutorando em Farmacologia pela Universidade Federal do Ceará (UFC), Fortaleza, Ceará, Brasil. 5 Fisioterapeuta, doutoranda em Ciências Médicas pela Universidade Federal do Ceará (UFC), coordenadora da Residência Integrada Multiprofissional em Atenção Hospitalar da UFC, Fortaleza, Ceará, Brasil. 6 Doutor em Pneumologia, Universidade Federal de São Paulo (UNIFESP), professor associado de Terapia Intensiva e Pneumologia do Departamento de Medicina Clínica, Universidade Federal do Ceará (UFC), Fortaleza, Ceará, Brasil.

\section{RESUMO}

Objetivo: analisar o conhecimento dos profissionais de saúde a respeito do uso da oxigenoterapia. Métodos: estudo quantitativo e transversal realizado em um Hospital Universitário da cidade de Fortaleza-CE. Participaram do estudo médicos, enfermeiros e fisioterapeutas. Para a coleta de dados utilizou-se um questionário semiestruturado contendo dados a respeito da formação acadêmica, tempo de experiência e conhecimento acerca da oxigenoterapia. Resultados: a amostra foi composta por 50 profissionais, com média de idade de $28,1 \pm 10,9$ anos, em sua maioria mulheres $80 \%(\mathrm{n}=40)$. Com relação à profissão, $44 \%(\mathrm{n}=22)$ possuem graduação em enfermagem, seguidas pela fisioterapia $32 \%(n=16)$ e medicina $22 \%(n=11)$. As indicações da oxigenoterapia com significância estatística são: hipoxemia, dessaturação e doença respiratória. Doença pulmonar obstrutiva crônica (DPOC), pneumotórax e hiperoxemia foram pontuadas como principais contraindicações, em relação aos efeitos adversos os mais prevalentes foram intoxicação, ressecamento e hipercapnia. O catéter nasal, máscara de Venturi, máscara com reservatório e macronebulização foram os tipos de oferta de oxigênio $\left(\mathrm{O}_{2}\right)$ mais pontuados $(\mathrm{p}<0,05)$. Conclusão: diante do exposto observamos que pode haver déficit em relação ao conhecimento dos profissionais de saúde a respeito do uso de oxigênio suplementar.

Palavras-chave: Oxigenoterapia. Conhecimento. Pessoal técnico de sáude.

\section{ABSTRACT}

Objective: To evaluate the knowledge of health professionals about the use of oxygen therapy. Methods: Quantitative and crosssectional study conducted in a university hospital in the city of Fortaleza-CE. Doctors, nurses and physiotherapists participated in the study. For data collection was used a semi-structured questionnaire with data about the academic background, long experience and knowledge of the oxygen therapy. Results: The sample consisted of 50 professionals, with a mean age of $28.1 \pm 10.9$ years, mostly women $80 \%(n=40)$. With regard to the profession, $44 \%(n=22)$ have a degree in nursing, physical therapy followed by $32 \%(n=16)$ and medicine $22 \%(n=11)$. The indications of oxygen therapy with statistical significance are: hypoxemia, desaturation and respiratory disease. Chronic obstructive pulmonary disease (COPD), pneumothorax and hyperoxia were scored as main contraindications, in relation to the adverse effects were the most prevalent intoxication, dryness and hypercapnia. The nasal catheter, mask Venturi mask with reservoir and macronebulização were the types of oxygen delivery $\left(\mathrm{O}_{2}\right)$ scorers $(\mathrm{p}<0.05)$. Conclusion: Given the above we note that there may be a deficit in relation to the knowledge of health professionals about the use of supplemental oxygen.

Keywords: Oxigen inhalation therapy. Knowledge. Allied health personnel.

Autor correspondente: Patriciane Hedwiges Barreto, Rua Guilherme Mendes, 616, Jardim Iracema, Fortaleza, Ceará. CEP: 60345-090. Telefone: +55 85 99249-2470. E-mail: patricianebarreto@hotmail.com

Conflito de interesses: Não há qualquer conflito de interesses por parte de qualquer um dos autores.

Recebido em: 16 Out 2016; Revisado em: 13 Jan 2017; Aceito em: 20 Jan 2017. 


\section{INTRODUÇÃO}

O oxigênio teve seu caráter vital reconhecido desde a sua descoberta, realizada de forma independente por Schelee, em 1772, e por Pristly, em 1774. Já em 1780, foi utilizado com propósitos médicos por Chaussier, que empregou o oxigênio em recém-nascidos com dificuldade respiratória. ${ }^{1}$ Desde então, a administração de oxigênio $\left(\mathrm{O}_{2}\right)$ vem sendo uma das mais importantes modalidades de terapia para pacientes com hipóxia resultante de condições comuns como infecções do trato respiratório inferior que, quando graves, podem levar à morte ou a sequelas em qualquer idade.,3

A oxigenoterapia é utilizada com o objetivo de manter valores da pressão parcial de oxigênio $\left(\mathrm{PaO}_{2}\right)$ adequadas, favorecendo o aumento da concentração desse gás no ar inspirado através da oferta de oxigênio suplementar. ${ }^{4}$ Está indicada para pacientes que em ar ambiente apresentam uma redução da $\mathrm{PaO}_{2}<60$ mmHg ou saturação periférica de oxigênio $\left(\mathrm{SpO}_{2}\right)<90 \%$, infarto agudo do miocárdio (IAM), $\mathrm{SpO}_{2}<88 \%$ em portadores de doenças cardiorrespiratórias durante a realização de exercício ou na deambulação e durante o sono, bem como nos casos de intoxicação por gases. ${ }^{5}$

A administração de oxigênio pode ser feita através dos sistemas de baixo ou de alto fluxo, de acordo com objetivo previsto para cada paciente. O sistema de baixo fluxo apresenta variação do fluxo, sua fração inspirada de $\mathrm{O}_{2}\left(\mathrm{FiO}_{2}\right)$ será determinada por diversos fatores tais como a quantidade de gás entregue, tipo de interface, bem como anatomia de cada indivíduo. O sistema de alto fluxo entrega um fluxo fixo, ou seja, $\mathrm{FiO}_{2}$ é constante. ${ }^{4}$

Os principais dispositivos utilizados para a terapêutica de oxigênio suplementar são os cateteres nasais; máscara simples e máscaras com reservatório que podem permitir ou não a reinalação. ${ }^{6}$

Apesar de ser essencial à vida, o oxigênio, como qualquer medicamento, quando administrado de forma indevida, pode ser tóxico e causar sérios prejuízos clínicos. ${ }^{7}$ Altas concentrações podem produzir lesão pulmonar e em outros tecidos, com a maior produção de radicais livres, bem como os efeitos da hiperóxia na distribuição da perfusão tecidual. ${ }^{8}$ Porém, a toxicidade, depende de fatores como pressão absoluta de oxigênio oferecido, duração a exposição e sensibilidade individual. ${ }^{9}$

Há cada vez mais evidências de que a hiperoxemia pode ser prejudicial em algumas condições clínicas, tais como: acidente vascular encefálico, infarto agudo do miocárdio, prematuridade extrema, doença pulmonar obstrutiva crônica (DPOC) e após a reanimação cardíaca extrapolar. No paciente de trauma em geral é mais difícil, porém é plausível que a administração rotineira de oxigênio nem sempre seja benéfica. ${ }^{10}$

\section{DEFINIÇÃO DE BOAS PRÁTICAS E ADEQUAÇÃO EM OXIGENOTERAPIA}

\section{Indicação}

Oxigênio deve ser prescrito para atingir um alvo de saturação de $94-98 \%$ para a maioria dos pacientes gravemente doentes ou $88-92 \%$ para aqueles em risco de insuficiência respiratória hipercápnica. ${ }^{11}$

\section{Administração da oxigenoterapia nos diferentes dispositivos}

\section{- Cânula nasal}

Método simples, barato, e o mais comumente usado. Excelente nos casos de acentuação da dispneia, durante a alimentação ou tosse, ocasiões em que seria necessário remover a máscara facial, se esta fosse a forma de administração. Pode ocorrer secura ou sangramento da mucosa nasal após a utilização de fluxos altos. A umidificação só se faz necessária para fluxos maiores que $4 \mathrm{~L} / \mathrm{min}^{12}$

\section{- Máscaras de Venturi}

São dispositivos que ofertam níveis constantes de $\mathrm{FiO}_{2}$ e possuem orifícios laterais, os quais permitem entrada de $\mathrm{O}_{2}$ do ambiente, esse tipo pode fornecer de $24 \%$ até $50 \%$ de $\mathrm{O}_{2}{ }^{6}{ }^{2}$

\section{- Máscara reservatório}

Existem dois tipos de máscaras com reservatório, com reinalação e sem reinalação de $\mathrm{CO}_{2}$. As máscaras com reservatório que permitem reinalação alcançam uma $\mathrm{FiO}_{2}$ de 60 a $80 \%$ a 10 litros por minuto, máscaras que evitam reinalação podem alcançar $\mathrm{FiO}_{2}$ de 80 a $95 \%$, isso ocorre devido à válvulas unidirecionais. ${ }^{13}$

O fornecimento de oxigênio a 100\% através de máscara reservatório pode ser administrado antes da intubação endotraqueal para pré-oxigenação, com objetivo de favorecer o aumento da saturação de oxigênio da hemoglobina. ${ }^{14}$

\section{Monitorização}

\section{- Oximetria de pulso (tipo de oxímetro)}

A oximetria de pulso é considerada o melhor método para triagem de pacientes com hipoxemia. O oxímetro posicionado no dedo ou no lóbulo da orelha nos fornece, através da pele e por leitura imediata, a saturação arterial do oxigênio $\left(\mathrm{SpO}_{2}\right)$ no ato do exame. O oxímetro é bastante sensível e fidedigno, com erro estimado de 1 a $2 \%$, quando comparado com o obtido pela gasometria arterial. Existem situações clínicas que alteram sua leitura, a saber: icterícia, metahemoglobinemia, onicomicose, esclerodermia, perfusão tecidual inadequada, pele com pigmentação escura e artefato. Apesar desses inconvenientes, a oximetria de pulso é considerada método excelente. Além dessa sua indicação, podemos usá-la para titular o fluxo de oxigênio mais preciso em repouso, no exercício e durante o sono. ${ }^{12}$

\section{- Gasometria arterial (pré e pós)}

A presença de uma saturação periférica de oxigênio $\left(\mathrm{SpO}_{2}\right)$ normal nem sempre faz negar a necessidade de medidas de 
gases sanguíneos, porque a oximetria de pulso se manterá inalterada em um paciente com tensão de oxigênio normal. Medições de gases do sangue são, portanto, necessárias o mais cedo possível em todas as situações em que estas medições possam afetar os resultados dos pacientes. ${ }^{11}$

\section{- Prescrição (legível e completa)}

O oxigênio deve sempre ser prescrito em um designado documento, porém, em caso de emergência o oxigênio deve ser administrado primeiro e documentado mais tarde. ${ }^{11}$ Nas prescrições devem estar descritos a duração do tratamento, fluxo e $\mathrm{FiO}_{2}$ desejados de acordo com o quadro clínico do paciente. Administração sem prescrições ou prescrições incompletas estão relacionadas à falha terapêutica ou efeitos colaterais do uso do oxigênio. ${ }^{15}$

Estudos mostram que existe ausência de protocolos sobre o uso de $\mathrm{O}_{2}$ e isso se deve ao fato dessa terapia ainda ser iniciada, modificada ou interrompida pela equipe sem comunicar ao médico responsável. ${ }^{16}$

O oxigênio exige receita médica, pois se trata de uma droga e está sujeita a lei, sendo necessário portanto sua prescrição para utilização. A equipe deve ser treinada para a administração, e com isso evitar a incidência de mau uso. ${ }^{17}$

Os profissionais que estão diretamente ligados a esta terapêutica devem ter conhecimento sobre a mesma, principalmente indicações, contraindicações e seus efeitos adversos, pois o uso demasiado de oxigênio suplementar pode ter efeitos deletérios.

Este estudo objetiva analisar o conhecimento dos profissionais de saúde a respeito do uso da oxigenoterapia.

\section{MÉTODO}

Estudo quantitativo, descritivo, observacional e transversal realizado em um Hospital Universitário da cidade de Fortaleza-CE, no período de agosto a setembro de 2015, nas unidades que atendem as especialidades de pneumologia, clinica médica, cardiologia, infectologia, gastroenterologia, neurologia, cirurgia, Unidade de Terapia Intensiva e sala de recuperação pós-anestésica. Participaram do estudo 50 profissionais de saúde: médicos, enfermeiros e fisioterapeutas diretamente ligados à assistência. Foram excluídos do estudo os profissionais que não estavam ligados diretamente à assistência dos referidos pacientes e os que não responderam o questionário por completo.

A coleta de dados se deu em dois momentos. Primeiramente a pesquisadora explicou os objetivos da pesquisa e solicitou assinatura do termo de consentimento livre e esclarecido (TCLE), no segundo momento foi entregue um questionário semiestruturado contendo dados a respeito da formação acadêmica, tempo de experiência e conhecimento acerca da oxigenoterapia, tais como suas indicações, contraindicações, efeitos deletérios, dificuldades encontradas na administração e tipos de suporte mais utilizados.
O banco de dados foi digitado no programa Microsoft Office Excel $^{\circledR}$ 2007, sendo as análises realizadas pelo software Statistical Package for Social Science (SPSS ${ }^{\circledR}$ ) versão 20. Foram utilizados os testes qui-quadrado e t de Student, de acordo com a normalidade, como também, o coeficiente de correlação de Pearson, todos adotando como nível de significância o valor de $\mathrm{p}<0,05$.

A pesquisa seguiu os preceitos éticos da resolução $n^{\circ} 466 / 12$ do Ministério da Saúde, portanto, a fase de coleta iniciou-se somente após a aprovação do projeto pelo comitê de ética em pesquisa em 27/07/2015 sob o número 1.159 .872 e mediante assinatura do TCLE.

\section{RESULTADOS}

A amostra foi composta por 50 profissionais, com média de idade de 28,1 $\pm 10,9$ anos, sendo $80 \%(\mathrm{n}=40)$ do gênero feminino. Em relação à profissão, 44\% $(n=22)$ possuem graduação em enfermagem, seguidas pela fisioterapia 32\% (n =16) e medicina $22 \%(\mathrm{n}=11)($ Tabela 1$)$.

Em resposta a quais seriam as principais indicações da oxigenoterapia os profissionais pontuaram principalmente a hipoxemia, dessaturação e doença respiratória (Tabela 2). Quanto às contraindicações da oxigenoterapia, a DPOC, o pneumotórax e a hiperoxemia apresentaram significância estatística (Tabela 3), em relação aos efeitos adversos os mais prevalentes foram intoxicação, ressecamento e hipercapnia (Tabela 4).

Tabela 1. Dados sociodemográficos dos profissionais do Hospital Universitário Fortaleza/CE.

\begin{tabular}{lcc}
\hline & N & $\%$ \\
\hline Profissão & 22 & 44 \\
Enfermagem & 16 & 32 \\
Fisioterapia & 11 & 22 \\
Medicina & 1 & 2 \\
Não informado & 5 & 10 \\
Formação prévia & 13 & 26 \\
Curso de Pneumologia & 16 & 32 \\
Curso de VM & 10 & 20 \\
Curso de TI & 7 & 14 \\
ACLS & 2 & 4 \\
RES médica & 5 & \\
RES multiprofissional &
\end{tabular}

*DP = Desvio padrão, VM = Ventilação mecânica, TI = Terapia Intensiva, ACLS = Suporte Avançado de Vida em Cardiologia, RES $=$ Residência. 
Tabela 2. Indicações da oxigenoterapia citadas pelos profissionais do Hospital Universitário Fortaleza/CE.

\begin{tabular}{lcc}
\hline \multicolumn{1}{c}{ Indicações } & N & \% \\
\hline Cefaleia & 3 & 6 \\
\hline Cianose & $5^{\#}$ & 10 \\
\hline Desconforto respiratório & $10^{\#}$ & 20 \\
\hline Dessaturação & $23^{\#}$ & 46 \\
\hline Dispneia & $13^{\#}$ & 16 \\
\hline Doença respiratória & $18^{\#}$ & 36 \\
\hline Edema Agudo de Pulmão & 3 & 6 \\
\hline Hipoxemia & $38^{\#}$ & 76 \\
\hline Infarto Agudo do Miocárdio & $13^{\#}$ & 26 \\
\hline Intoxicação & $5^{\#}$ & 10 \\
\hline Intubação & $4^{\#}$ & 8 \\
\hline IRpA & $13^{\#}$ & 26 \\
\hline PCR & $7^{\#}$ & 14 \\
\hline Pós-operatório & 3 & 6 \\
\hline Taquipneia & $4^{\#}$ & 8 \\
\hline Outras & 25 & 50 \\
\hline
\end{tabular}

${ }^{\#} \mathrm{p}<0,05 ;$ Teste $\mathrm{t}$ de Student.

Tabela 3. Contraindicações da oxigenoterapia citadas pelos profissionais do Hospital Universitário Fortaleza/CE.

\begin{tabular}{lcc}
\hline \multicolumn{1}{c}{ Contraindicações } & N & \% \\
\hline Atelectasia grave & 2 & 4 \\
Desnecessário & $7^{\#}$ & 14 \\
\hline DPOC & $30^{\#}$ & 60 \\
Hipercapnia & 2 & 4 \\
\hline Hiperoxemia & $11^{\#}$ & 22 \\
\hline Intolerância do paciente & 2 & 4 \\
Pneumotórax & $7^{\#}$ & 14 \\
Outras & 13 & 26 \\
\hline
\end{tabular}

${ }^{\#} \mathrm{p}<0,05 ;$ Teste $\mathrm{t}$ de Student.

Tabela 4. Efeitos adversos da oxigenoterapia citados pelos profissionais do Hospital Universitário Fortaleza/CE.

\begin{tabular}{lll}
\hline \multicolumn{1}{c}{ Efeitos Adversos } & N & \% \\
\hline Atelectasia & $9^{\#}$ & 18 \\
\hline Depressão respiratória & $8^{\#}$ & 16 \\
\hline Estresse oxidativo & $9^{\#}$ & 18 \\
\hline Hipercapnia & $11^{\#}$ & 22 \\
\hline Hiperóxia & $10^{\#}$ & 20 \\
\hline Intoxicação & $14^{\#}$ & 28 \\
\hline Lesões do endotélio & $6^{\#}$ & 12 \\
\hline Narcose & $10^{\#}$ & 20 \\
\hline Ressecamento & $12^{\#}$ & 24 \\
\hline Retinopatia & $5^{\#}$ & 10 \\
Sonolência & $4^{\#}$ & 8 \\
Vasoconstricção & $6^{\#}$ & 12 \\
Outros & 20 & 40 \\
\hline
\end{tabular}

${ }^{\#} \mathrm{p}<0,05 ;$ Teste t de Student.
Dentre os profissionais médicos que participaram da pesquisa $33 \%(n=3)$ afirmaram que tem o hábito de prescrever oxigênio, $44 \%(n=4)$ pontuaram que não e $44 \%(n=4)$ afirmaram que prescrevem às vezes. Não houve correlação entre os profissionais médicos que prescrevem oxigenoterapia e os que solicitam o monitoramento por gasometria arterial $(\mathrm{r}=0,12$; correlação de Pearson; $\mathrm{p}=0,8$ teste de qui-quadrado).

Quando indagados sobre o acompanhamento de pacientes em oxigenoterapia, 64\% $(n=31)$ da amostra afirmaram que possuem o hábito de verificar se o $\mathrm{O}_{2}$ está na prescrição do paciente e $80 \%(n=44)$ solicitam que pacientes em oxigenoterapia sejam monitorizados com oximetria de pulso. Não ocorreu diferença estatística nas respostas $(\mathrm{p}=0,052$; teste de qui-quadrado) em relação às principais dificuldades encontradas na administração de oxigênio (Tabela 5).

Em relação aos tipos de oferta de $\mathrm{O}_{2}$ que conhecem, ocorreu uma diferença significativa ( $\mathrm{p}<0,05$; teste $\mathrm{t}$ de Student) quando comparado às formas de administração, onde $23,1 \%$ afirmaram conhecer tanto o cateter nasal como a máscara com reservatório e $22,7 \%$ destacaram que conhecem a máscara de Venturi e também a macronebulização como formas de oferta de $\mathrm{O}_{2}$ para o paciente (Tabela 6).

Tabela 5. Dificuldades encontradas na administração de oxigênio citadas pelos profissionais do Hospital Universitário Fortaleza/CE.

\begin{tabular}{lcc}
\hline \multicolumn{1}{c}{ Dificuldades } & n & \% \\
\hline Falta de gasometria & 10 & 13,5 \\
\hline Falta de oximetria & 10 & 13,5 \\
Material inadequado & 24 & 32,4 \\
Pouca cooperação do paciente & 13 & 17,6 \\
Treinamento inadequado da equipe & 17 & 23 \\
\hline
\end{tabular}

Tabela 6. Tipos de oferta de $\mathrm{O}_{2}$ segundo os profissionais do Hospital Universitário Fortaleza/CE.

\begin{tabular}{lcc}
\hline \multicolumn{1}{c}{ Tipos } & n & \% \\
\hline Máscara de Venturi & 49 & 22,7 \\
Cateter nasal & 50 & 23,1 \\
Macronebulização & 49 & 22,7 \\
Máscara com Reservatório & 50 & 23,1 \\
Alto fluxo nasal & 18 & 8,3 \\
Total & $\mathbf{2 1 6}$ & $\mathbf{1 0 0 , 0}$ \\
\hline
\end{tabular}

${ }^{*} \mathrm{p}<0,05 ;$ Teste $\mathrm{t}$ de Student.

\section{DISCUSSÃO}

No presente estudo os profissionais pontuaram a hipoxemia como a principal indicação para oxigenoterapia, corrobora com o presente estudo a British Thoracic Society (BTS), a qual pontua como principais indicações do uso do oxigênio suplementar a correção da hipoxemia grave devido às suas repercussões no organismo, prevenção de hipoxemia em pacientes debilitados e também no alívio do desconforto respiratório. De acordo com a 
BTS há grupos de riscos para a utilização do oxigênio suplementar, dentre eles estão: insuficiência respiratória hipercápnica, hipoxemia leve a moderada em pacientes com acidente vascular cerebral (AVC), aspiração de ácidos, dentre outros. ${ }^{11}$

A oxigenoterapia é benéfica, porém, seu uso indiscriminado pode propiciar efeitos adversos. Um estudo holandês observou que valores de $\mathrm{PaO}_{2}$ alcançados em pacientes de UTI são maiores que os recomendados na literatura. Valores altos de $\mathrm{FiO}_{2}$, bem como valores baixos e altos de $\mathrm{PaO}_{2}$ estão associados com a mortalidade intra-hospitalar em pacientes de UTI. A exposição a altas concentrações de oxigênio por tempo prolongado pode causar dano ao epitélio brônquico com aparecimento de bronquite, diminuição da atividade ciliar e da função bactericida bronquial, hipercapnia, hiperóxia e atelectasias por reabsorção. ${ }^{18}$

A oximetria de pulso pode garantir o uso mais eficiente da oxigenoterapia. ${ }^{19}$ Estudos pontuam correlação significativa entre $\mathrm{SpO}_{2}$ e $\mathrm{SaO}_{2}$, demonstrando que a utilização do oxímetro pode ser segura e eficaz na avaliação do paciente em oxigenoterapia, entretanto, podem ocorrer interferências no oxímetro e a confirmação dos valores pode ser feita através da gasometria arterial. ${ }^{5}$

Em um estudo multicêntrico de oxigenoterapia no qual foi realizada uma auditoria nacional aos procedimentos, a ausência da duração e monitorização da oxigenoterapia foram os erros mais frequentes encontrados. Dentre os pacientes incluídos no estudo, $173(29,1 \%)$ apresentaram monitorização e 422 (70,9\%) estavam sem monitorização. ${ }^{15}$ No presente estudo $80 \%$ responderam que solicitam a monitorização do paciente em uso de oxigênio, sendo este um fator imprescindível para a otimização desta terapêutica.

A adequação no uso de dispositivos utilizados para oferta de oxigênio e a monitorização adequada ainda tem apresentado alguns déficits. No estudo realizado em Santa Catarina com 33 pacientes em uso de oxigênio, apenas $51,6 \%$ estavam com os valores de $\mathrm{PaO}_{2}$ adequados, a monitorização foi realizada por gasometria arterial e oximetria de pulso e correlação dos valores de saturação medidos. ${ }^{5}$

Ainda nesse estudo, observou-se que os dispositivos de oxigenoterapia mais utilizados pelos participantes foram a

\section{REFERÊNCIAS}

1.Saugstad OD. Is oxygen more toxic than currently believed? Pediatrics. 2001;108(5):1203-5.

2. Usen S, Webert M. Clinical signs of hypoxaemia in children with acute lower respiratory infection: indicators of oxygen therapy. Int $\mathrm{J}$ Tuberc Lung Dis. 2001;5(6):505-10.

3. Muhe L, Webert M. Oxygen delivery to children with hypoxaemia in small hospitals in developing countries. Int J Tuberc Lung Dis. 2001;5(6):527-32.

4. Pádua AI, Alvares F, Martinez JA. Insuficiência respiratória. Medicina (Ribeirão Preto). 2003;36:205-13. máscara de Venturi, cânula nasal e máscara com reservatório, entretanto, nenhum dos dispositivos apresentou a correlação $\mathrm{FiO}_{2}$ x Fluxo esperada. ${ }^{5}$ Nesta pesquisa a maioria dos profissionais afirmou conhecer cateter nasal e máscara com reservatório seguido de máscara de Venturi e macronebulização, sugerindo que estes possam ser os dispositivos mais utilizados em seus serviços.

A prescrição médica é imprescindível, assim como o manuseio e a monitorização adequada pela equipe multiprofissional, por se tratar de uma terapêutica medicamentosa, portanto, a equipe deve conhecer os tipos de dispositivos utilizados para oferta desta terapia, bem como indicações, contraindicações e seus efeitos adversos. ${ }^{20}$

Nos resultados desta pesquisa verificou-se que o número de médicos que prescrevem oxigênio ainda é pequeno quando comparado aos que não prescrevem, isso implica diretamente na segurança dos pacientes que fazem uso desse medicamento. Em um estudo desenvolvido no Hospital Waikato realizou-se uma auditoria para avaliar práticas de prescrição de oxigênio e o dano potencial de toxicidade nos pacientes sem prescrição adequada. Os autores verificaram que dos cento e vinte pacientes que receberam a terapia de oxigênio somente $51,7 \%$ apresentaram prescrições corretas de oxigênio, sugerindo que as prescrições atuais de oxigênio não são satisfatórias e podem favorecer risco de toxicidade do oxigênio nos doentes. ${ }^{21}$

\section{CONCLUSÃO}

Os resultados desse estudo evidenciaram que grande parte dos profissionais de saúde conhecem a respeito do uso de oxigênio suplementar, porém observou-se que um número significatico de participantes ainda apresentam deficiência em relação ao conhecimento. Isso pode ser devido a escassez de material adequado, falta de treinamento para os profissionais e pouca colaboração do paciente, favorecendo o uso de frações inspiradas de oxigênio e dispositivos inadequados, o que pode repercutir de forma negativa no quadro clínico dos pacientes.

A conscientização de todos os profissionais envolvidos na assistência direta ao paciente e a educação permanente sobre a importância da adequação do uso do oxigênio se fazem necessárias para uma intervenção eficaz.

5. Kock KS, Rocha PA, Silvestre JC, Coelho D, Leite KR. Adequações dos dispositivos de oxigenoterapia em enfermaria hospitalar avaliadas por oximetria de pulso e gasometria arterial. ASSOBRAFIR Ciência. 2014;5(1):53-64.

6. Lindahl SG. Oxygen and life on earth: an anesthesiologist's views on oxygen evolution, discovery, sensing and utilization. Anesthesiology. 2008;109(1):7-13.

7. MacIntyre NR. Supporting oxygenation in acute respiratory failure. Respir Care. 2013;58(1):142-50.

8. Roffe C, Ali K, Warusevitane A, Sills S, Pountain S, Allen M, et 
al. The SOS pilot study: a RCT of routine oxygen supplementation early after acute stroke-effect on recovery of neurological function at one week. PloS One. 2011;6(5):1-9.

9. Rudan I, Tomaskovic L, Boschi-Pinto C, Campbell H; WHO Child Health Epidemiology Reference Group. Global estimate of the incidence of clinical pneumonia among children under five years of age. Bull World Health Organ. 2004;82(12):895-903.

10. Mcmullan J, Rodrigues D, Hart KW, Lindsell CJ, Vonderschmidt k, Wayne B, et al. Prevalence of prehospital hypoxemia and oxygen use in trauma patients. Mil Med. 2013;178(10):1121-5.

11. O'Driscoll BR, Howard LS, Davison AG; British Thoracic Society. BTS guideline for emergency oxygen use in adult patients. Thorax. 2008;63(Suppl 6):1-68.

12. Sociedade brasileira de pneumologia e tisiologia (SBPT). Oxigenoterapia domiciliar prolongada. J. Pneumologia. 2000;26(6):341-50.

13. Chakrabarti B, Calverley PM. Management of acute ventilatory failure. Postgrad Med J. 2006;82(969):438-45.

14. Amantea SL, Piva JP, Rodrigues MI, Bruno F, Garcia PC. Acesso rápido à via aérea. Pediatria (Rio J.). 2003;79 (Suppl 2):S127-38.

15. Neves JT, Lobão MJ. Estudo multicêntrico de oxigenoterapia - uma auditoria nacional aos procedimentos de oxigenoterapia em enfermarias de medicina interna. Rev Port Pneumol. 2012;18(2):80-85.

16. Brokalaki H, Matziou V, Zyga S, Kapella M, Tsaras K, Brokolaki $\mathrm{E}$, et al. Omissions and errors during oxygen therapy of hospitalized patients in a large city of Greece. Intensive Crit Care Nurs. 2004;20(6):352-7.

17. Wong M, Elliott M. The use of medical orders in acute care oxygen therapy. Br J Nurs. 2009;18(8):462-4.

18. de Jonge E, Peelen L, Keijizers PJ, Joore H, de Lange D, van der Voort PH, et al. Association between administered oxygen, arterial partial oxygen pressure and mortality in mechanically ventilated intensive care unit patients. Crit Care. 2008;12(6):R156.

19. World Health Organization. Oximetria Projeto Global Pulse [Internet]. Genebra: WHO, 2010 [cited 2010 Oct 01]. Available from: http://www.who.int/patient segurança / safesurgery / pulse_oximetry / en / index.html

20. Castanheira NP, Valério MC, Weigert FC. Gerenciamento do consumo do oxigênio durante a inaloterapia: oportunidade para redução de custos através da capacitação dos profissionais. Revista Saúde e Desenvolvimento. 2014;5(3):9-30.

21. Holbourn A, Wong J. Oxygen prescribing practice at Waikato Hospital does not meet guideline recommendations. Intern Med J. 2014;44(12a):1231-4.

\section{Como citar:}

Barreto PH, Silva FS, Vasconcelos RS, Sales RP, Vasconcelos TB, Nogueira AN, et al. Análise do conhecimento dos profissionais de saúde sobre o uso de oxigenoterapia em um hospital universitário de Fortaleza-CE. Rev Med UFC. 2017 set-dez;57(3):18-23. 